\title{
Atherectomy of the Left Main Coronary Artery with Percutaneous Cardiopulmonary Bypass Support
}

\author{
David W.M. Muller, MBBS, Stephen G. Ellis, MD, and Eric J. Topol, MD
}

eft main (LM) coronary ar-tery stenoses have conventionally been precluded from percutaneous coronary angioplasty because of the prohibitive risk of irreversible hemodynamic collapse after acute closure of the artery, and a relatively high risk of late sudden death. ${ }^{1}$ When protected by left anterior descending and circumflex coronary artery bypass grafts, angioplasty of the LM coronary artery can be safely performed but is limited by a rate of recurrent stenosis in excess of $50 \% .^{2}$ Coronary atherectomy has recently been advocated as an alternative procedure because of preliminary data suggesting a lower incidence of acute

From the Division of Cardiology, Department of Internal Medicinc, University of Michigan Medical Center, B1 F245, 1500 East Medical Center Drive, Ann Arbor, Michigan 48109. Manuscript received January 30, 1989; revised manuscript received and accepted April $12,1989$.

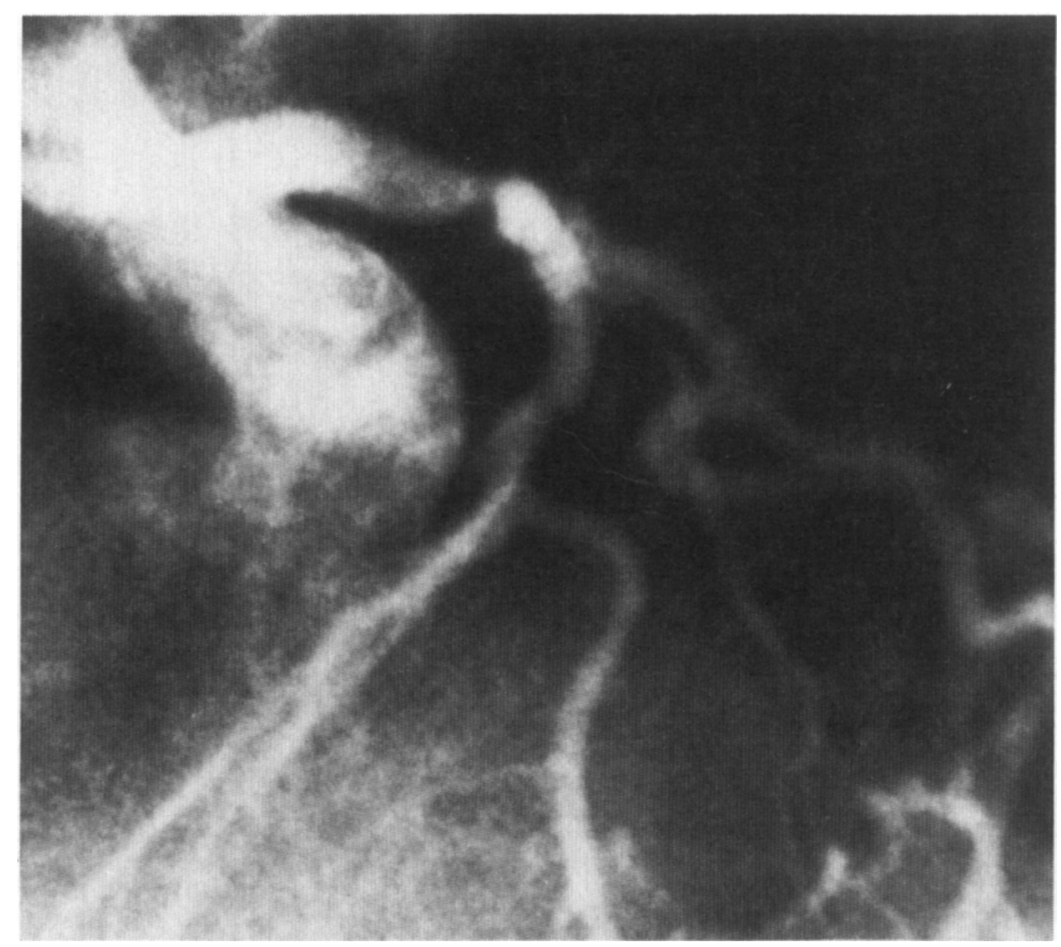

FIGURE 1. Single cineanglographic trame in the left anterior oblique projection before the atherectomy shows the distal left main coronary artery stenosis. ruary 1988 when he developed recur rent Canadian Heart Association class IV angina. An exercise thallium study demonstrated inferior, apical and posterior defects with redistribution consistent with ischemia. Coronary angiography showed a $70 \%$ distal LM coronary stenosis, complete occlusion of the midleft anterior descending and the proximal right coronary arteries and subtotal occlusion of the obtuse marginal branch of the circumflex coronary artery. The vein graft to the posterolateral branch of the right coronary artery was occluded but the remaining grafts were patent. Coronary angioplasties of the distal LM and obtuse marginal stenoses were successfully performed with good symptomatic response. Symptoms recurred, however, in September 1988. Repeat coronary angiography showed recurrence of the $L M$ and obtuse marginal branch stenoses, occlusion of the obtuse marginal and right coronary grafts and development of a long, irregular stenosis in the midleft anterior descending ar. tery distal to the insertion of the saphenous vein graft. Coronary angioplasties of the left anterior descending artery and obtuse marginal stenoses were then performed with a reduction in caliper-measured stenosis severity from 85 to $11 \%$ and 85 to $16 \%$, respectively. Despite the satisfactory angiographic results, no symptomatic improvement was apparent and he was readmitted in November 1988. Repeat angiography showed minimal residual stenosis at the sites of recent coronary angioplasty and no change in the severity of the $70 \%$ distal LM coronary stenosis (Figure 1). Although it would be incompletely protected by coronary bypass grafts, coronary atherectomy was the procedure preferred because of the early recurrent stenosis after balloon angioplasty and the patient's desire to avoid a second surgical procedure.

To provide optimal hemodynamic support during the procedure, cardiopulmonary bypass was initially instituted using the Bard cardiopulmonary support device. Right femoral arterial $(20 \mathrm{Fr})$ and venous $(22$ 
Fr) cannulas were inserted percutaneously under local anesthetic. Commencement of bypass resulted in transient systemic hypotension, which was readily corrected by the administration of additional fluids through the support system. Thereafter, systemic systolic pressure remained between 85 and $110 \mathrm{~mm} \mathrm{Hg}$ with bypass flow rates of 3.5 to 4.5 liters/min. A total of 30,000 U of heparin was administered intravenously and partial thromboplastin time was monitored throughout the procedure. Atherectomy of the distal LM stenosis was then performed through an 11 Fr left coronary guiding catheter advanced from the left femoral artery. A 7 Fr Simpson directional atherectomy device (Figure 2) was used to remove 12 cores of tissue. Placement of the device resulted in $2 \mathrm{~mm}$ of ST-segment elevation in monitored leads $I I$ and $V_{5}$ commencing soon after left main occlusion and lasting throughout each pass of the device. These electrocardiographic changes were not associated with chest pain or hypotension and resolved rapidly after removal of the catheter. After minimal residual stenosis was demonstrated (Fig. ure 3), the atherectomy catheter was removed, the tissue obtained sent for histologic examination and the support system access site surgically closed. The postoperative course was uneventful apart from the need for a 9-unit blood transfusion due to

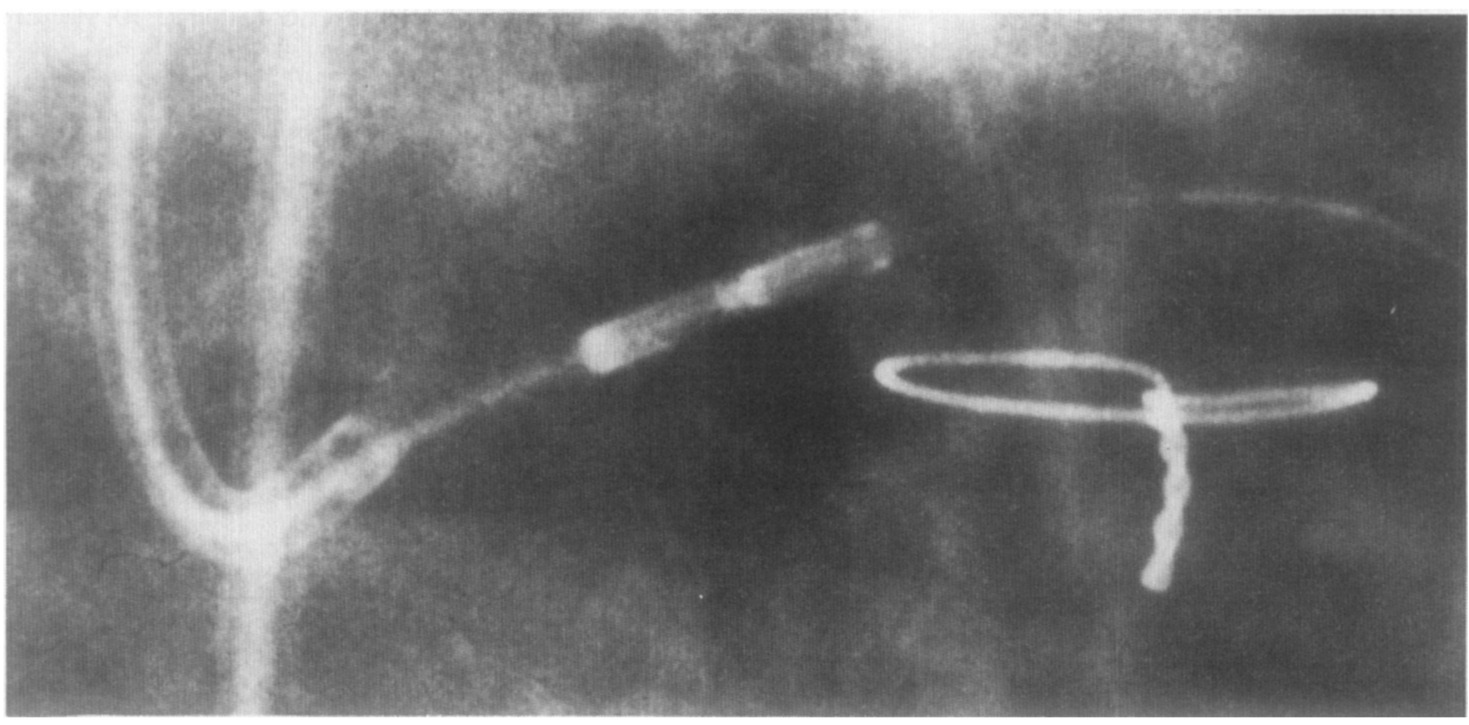

FIGURE 2. Right anterior oblique projection shows the atherectomy device positioned within the left main coronary artery.

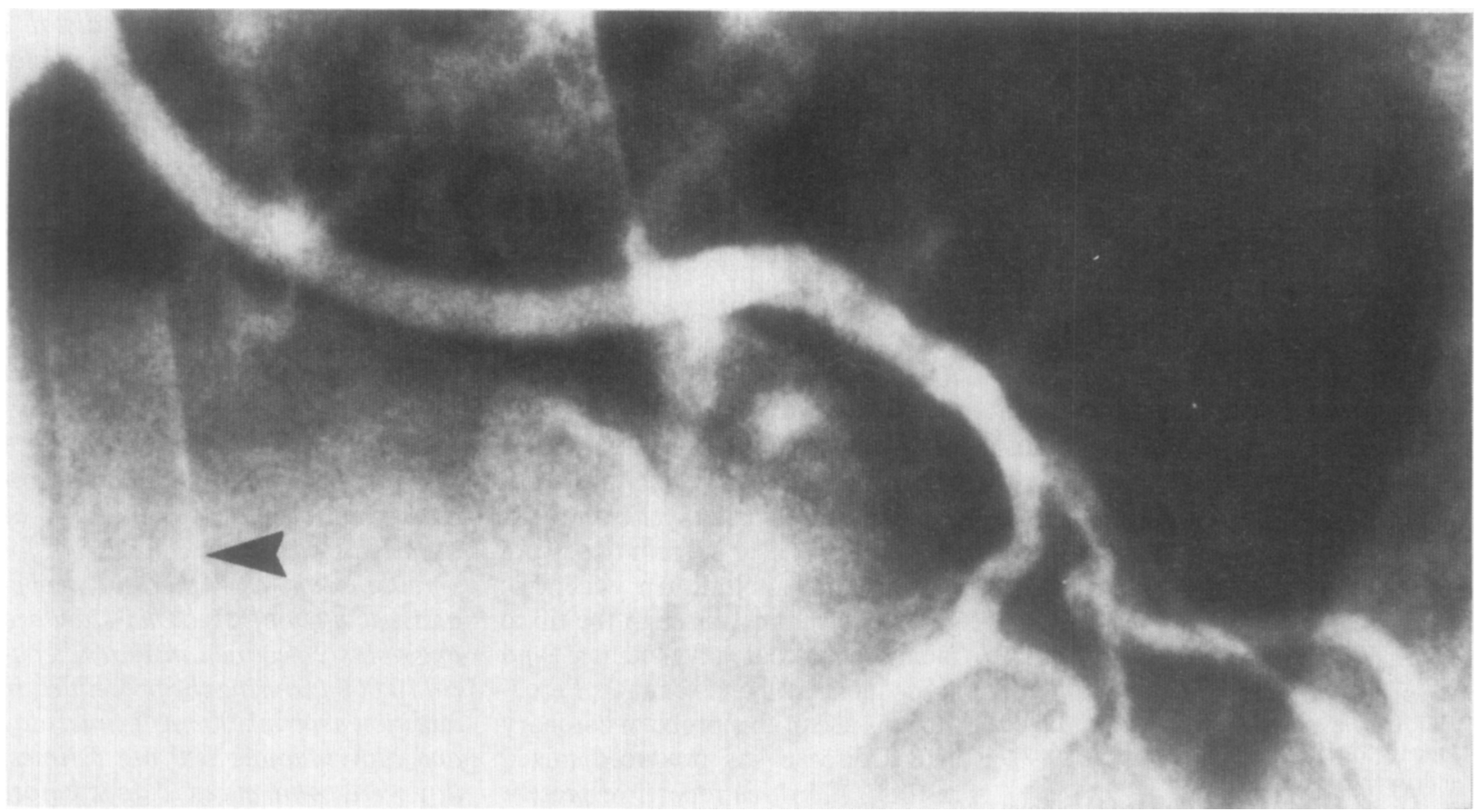

FICURE 3. Cineangiogram of the left main coronary artery after successful atherectomy shows minimal residual stenosis. The 22 Fr percutaneous cardiopulmonary bypass cannula lying within the right atrium is indicated by the arrow. 
losses within the support system and perioperative access site hemorrhage. A predischarge exercise thallium study showed a small fixed apical defect but no reversible ischemia. The patient was discharged 7 days postoperatively receiving coumadin and diltiazem and has remained asymptomatic with no evidence of provocable ischemia during the 5-month follow-up period.

Technological advances in dilatation catheter and guidewire design have greatly facilitated complex coronary angioplasty. Unprotected balloon angioplasty of the LM coronary artery, however, has remained relatively contraindicated because of the risk of acute closure and cardiogenic shock and the inability to perform prolonged balloon inflations. Several alternatives ${ }^{5}$ are being evaluated to reduce the severity of myocardial ischemia during LM coronary interventions and may reduce the likelihood of restenosis. During conventional balloon angioplasty, myocardial ischemia may be minimized directly using an autoperfusion bal- loon catheter or by infusing fluorinated hydrocarbons into the distal coronary circulation through the central lumen of the dilatation catheter. Alternatively, the use of coronary sinus retroperfusion techniques, percutaneous cardiopulmonary support or intraaortic balloon counterpulsation may indirectly reduce myocardial ischemia. Although these strategies may permit prolonged balloon inflations, this has not yet been shown to reduce the incidence of intimal dissection or restenosis.

Two promising alternatives are being investigated. Coronary atherectomy, first reported by Simpson et al, ${ }^{6}$ has been successfully used in the LM coronary artery and preliminary data from peripheral vascular procedures suggest that the recurrence rate may be significantly reduced after this procedure. Adequate longterm follow-up studies, however, have not yet been completed. Second, a variety of endoluminal stents have been developed but, to date, these have not been deployed in the LM coronary artery. In this patient, the combined use of 2 of these emerging strategies allowed thorough removal of atheromatous plaque in the LM coronary artery. Although experience with each device is limited and long-term follow up incomplete, this combination of new technologies appears to offer the potential for the expansion of the indications for percutaneous coronary intervention, obviating the need for coronary surgery in selected high risk situations.

1. Hartzler GO, Rutherford BD, McConahay DR Johnson WL, Giorgi LV, Calkins M, Lignon R. Left main coronary angioplasty-a caution (abstr). JACC 1988;11 (suppl 2):61A.

2. Demopolous P, Selmon M, Simpson J, McAuley B. Percutaneous balloon angioplasty of left main coronary stenoses-acute and long term results (abstr). JACC 1988;11 (suppl 2):61A.

3. Selmon MR, Robertson GC, Simpson JB. Rcstenosis in peripheral transluminal atherectomy (abstr). Circulation 1988,(suppl In 78:II-269.

4. Vogel RA. The Maryland experience: angioplasty and valvuloplasty using percutaneous cardiopulmonary support. Am J Cardiol 1988,62:11 K-14K

5. Topol EJ. Emerging strategies for failed percutaneous transluminal coronary angioplasty. Am J Cardiol 1989.63:249-250.

6. Simpson JB, Robertson GC, Selmon MR. Percutaneous coronary atherectomy (abstr). Circulation 1988,(suppl II)78:II-82.

\section{Coronary Artery Thermal Damage During Percutaneous "Hot Tip" Laser-Assisted Angioplasty}

Eric Rosenthal, MB, ChB, MRCP, Joseph K. Montarello, MB, BS, FRACP, Tim Palmer, MB, BS, MRCPathol, and Paul V.L. Curry, MD

A major postulated advantage of laser angioplasty as an alternative treatment for occlusive vascular disease is its ability to vaporize obstructive atheroma. ${ }^{1,2}$ The bare fiber systems used initially in the peripheral vasculature were able to traverse occlusions but the channels produced were small, subsequent balloon dilatation was always necessary and a high perforation rate was noted. ${ }^{3}$ The "hot tip" laser thermal probe produced by encasing the bare fiber tip

From the Department of Cardiology, Guy's Hospital, St. Thomas Street, London, SE1 9RT United Kingdom. Manuscript received January 31, 1989; revised manuscript received and accepted April 4, 1989.

Dr. Montarello was supported by a gran from the Lions Heart Research Foundation of South Australia. in a metal cap 4 overcame some of these limitations. The relatively large, smooth and oval metal cap enhanced coaxial placement while localization of all the laser energy (by heat conversion) at the tip made perforations infrequent. ${ }^{5,6}$ In the periphery this device recanalized lesions previously impassable to a guidewire and balloon system. ${ }^{7}$ For intracoronary use the smaller peripheral artery probes ( 1.5 to $1.7 \mathrm{~mm}$ tips) were modified to include an eccentric guidewire channel through the tip to facilitate coaxial advancement and subsequent balloon exchange. Percutaneous use of this probe in coronary artery disease has produced mixed results. ${ }^{8-10}$ In this report we present experience using a more flexible laser thermal device.
We used a laser probe comprised of a $116 \mu$ core optical fiber contained in a soft polyurethane catheter (Trimedyne Inc.). The distal 2 $\mathrm{cm}$ of the fiber, between the catheter and the 1.7-mm diameter tip, was enclosed in a floppy spiral metal coil that further enhanced the probe flexibility. A guidewire channel passed up the length of the catheter and through the center of the tip allowing it to be advanced over $a$ 0.014-inch guidewire. An Optilase argon laser (Trimedyne Inc.) set at 10 watts ( $>80 \%$ coupling efficiency) was used to heat the tip.

Patients in whom balloon angioplasty had been or was likely to be unsatisfactory were considered for percutaneous laser thermal probeassisted angioplasty. Our standard angioplasty regimen includes heparin 10,000 Uduring the procedure; in addition aspirin $300 \mathrm{mg} 3$ times/day and dipyridamole $100 \mathrm{mg} 3$ times/ day were commenced 2 days before the procedure. A temporary pacing electrode was placed in the right ven- 